\title{
Relationship between airway narrowing, patchy ventilation and lung mechanics in asthmatics
}

\author{
N.T. Tgavalekos*,\#, G. Musch*, R.S. Harris ${ }^{\star},+$, M.F. Vidal Melo*, T. Winkler*, \\ T. Schroeder*, R. Callahan*, K.R. Lutchen\# and J.G. Venegas*
}

ABSTRACT: Bronchoconstriction in asthma results in patchy ventilation forming ventilation defects (VDefs). Patchy ventilation is clinically important because it affects obstructive symptoms and impairs both gas exchange and the distribution of inhaled medications. The current study combined functional imaging, oscillatory mechanics and theoretical modelling to test whether the degrees of constriction of airways feeding those units outside VDefs were related to the extent of VDefs in bronchoconstricted asthmatic subjects.

Positron emission tomography was used to quantify the regional distribution of ventilation and oscillatory mechanics were measured in asthmatic subjects before and after bronchoconstriction. For each subject, ventilation data was mapped into an anatomically based lung model that was used to evaluate whether airway constriction patterns, consistent with the imaging data, were capable of matching the measured changes in airflow obstruction.

The degree and heterogeneity of constriction of the airways feeding alveolar units outside VDefs was similar among the subjects studied despite large inter-subject variability in airflow obstruction and the extent of the ventilation defects. Analysis of the data amongst the subjects showed an inverse relationship between the reduction in mean airway conductance, measured in the breathing frequency range during bronchoconstriction, and the fraction of lung involved in ventilation defects.

The current data supports the concept that patchy ventilation is an expression of the integrated system and not just the sum of independent responses of individual airways.

KEYWORDS: Airway obstruction, constriction, elastance, heterogeneous, oscillatory, resistance

$\mathrm{n}$ asthma, ventilation distribution and lung mechanics are extensively affected by heterogeneous airway constriction. Detailed imaging studies in the bronchoconstricted lung using magnetic resonance imaging [1, 2] and positron emission tomography (PET) [3, 4] have demonstrated the presence of patchy ventilation characterised by large ventilation defects (VDefs). The extent of these VDefs initially led investigators to speculate that they were likely to be caused by severe constriction of central airways feeding the constricted regions. However, experimental evidence and theoretical modelling suggest that most of the functional impairment in asthma is caused by heterogeneous constriction of peripheral airways [5]. This mechanical evidence is thus inconsistent with the concept that constriction of large airways could be exclusively responsible for VDefs. However, the current authors have recently demonstrated that even a symmetric airway tree undergoing uniform smooth muscle activation can develop highly heterogeneous ventilation as a result of severely constricted peripheral airways clustered in VDefs similar to those observed with imaging [3,4]. Furthermore, this model predicted that, for constrictive stimuli beyond a critical level, airway narrowing did not progress throughout the entire airway tree but was localised, increasing the extent of the lung covered by VDefs.

The latter prediction cannot be directly tested experimentally because limited spatial resolution of current imaging techniques does not allow visualisation of all the affected airways in a breathing lung. However, in a recent study the present authors showed that combining PET imaging with measurements and analysis of the frequency dependence of dynamic resistance $(R L)$ and elastance $(E \mathrm{~L})$, could be used to test hypothetical airway constriction patterns imposed on an anatomically consistent model of the airway tree [6].
AFFILIATIONS

*Depts of Anesthesia and Critical Care,

Medicine (Pulmonary and Critical

Care Unit and Clinical Epidemiology Unit), Massachusetts General Hospital,

\#Dept of Biomedical Engineering, Boston University and,

+Harvard Medical School, Boston, MA, USA

CORRESPONDENCE

J.G. Venegas

Dept of Anesthesia and Critical Care (CLN-237F)

Massachusetts General Hospital Boston

02114 MA

USA

E-mail: jvenegas@partners.org

Received:

August 292006

Accepted after revision:

February 282007

STATEMENT OF INTEREST

None declared.

European Respiratory Journa

Print ISSN 0903-1936

Online ISSN 1399-3003 
These image-guided modelling tools are used in the present study to evaluate the relationship between obstructive changes of the airway tree and relative extent (size) of the VDefs in bronchoconstricted asthmatics.

For this purpose, VDefs defined from PET images of each subject were mapped into the corresponding acini of an anatomically consistent three-dimensional (3-D) model of the airway tree [7]. Bronchoconstriction was simulated by systematically varying the lumen of specific airways of the tree. This allowed the definition of spatial patterns of airway obstruction that best approached the changes in oscillatory mechanics after bronchoprovocation in each of the subjects. The spatial patterns were evaluated to test whether constriction of airways feeding those units outside the VDefs could be associated with the differences, observed amongst subjects, in the extent of the lung covered by VDefs.

\section{METHODS}

A group of 10 mild-to-moderate asthmatic subjects were studied before and after bronchoconstriction with a protocol approved by the Massachusetts General Hospital review board (Boston, MA, USA; table 1). After the subjects were positioned in a supine manner in the PET scanner, oscillatory lung mechanics [8,9] and PET scans $[4,10]$ were acquired within $5 \mathrm{~min}$ of each other. Baseline data were acquired in the following order: 1) transmission PET scan and oscillatory mechanics; 2) emission PET scan of i.v. injected ${ }^{13} \mathrm{NN}$-saline during apnoea (30 s) followed by a 3-min washout with normal breathing; and 3) spirometry. Whilst in the scanner, a standard five-breath methacholine challenge was administered at a dose previously established to decrease the subject's upright provocation dose causing a $20 \%$ fall in forced expiratory volume in one second [11]. At 5-10 min following the challenge, another set of imaging and mechanics data was collected. VDefs were defined, from the end of the washout PET scan during bronchoconstriction, as sets of contiguous voxels with $\geqslant 20 \%$ of the highest residual tracer concentration. A 20\% threshold for residual tracer concentration was selected because at thresholds between 20 and 30\%, the change in size of VDefs was found to be rather insensitive to the threshold level selected. In addition, a constant $20 \%$ threshold for all subjects yielded the largest possible VDefs size within that range of thresholds.

Oscillatory mechanics were assessed by delivering, through a mouthpiece, seven superimposed sinusoidal waveforms with frequencies ranging $0.15-8 \mathrm{~Hz}$. Transpulmonary pressure $\left(P_{\mathrm{tp}}\right)$, measured as the difference between airway opening pressure and oesophageal pressure, and flow (Qao) at the airway opening measured with a pneuomotachograph, were electronically acquired and monitored for 2-3 min. Signal processing on $Q$ ao and $P_{\mathrm{tp}}$ was used to estimate values of $R \mathrm{~L}$ and $E \mathrm{~L}$ as a function of frequency [8].

\section{Computational modelling}

A 3-D model of the airway tree was used to simulate $R \mathrm{~L}$ and $E \mathrm{~L}$ for a set of assumed airway dimensions and tissue structural properties as previously described [7]. The impedance of a single airway is calculated using an acoustic transmission line model with a resistive and inertial element. Each airway also includes a shunt airway wall compliance, which is modelled as the parallel combination of soft tissue and cartilage. The airway wall properties are a function of airway diameter and airway wall thickness [12]. For each subject studied, the model's total gas volume was scaled to match the subject's plethysmographically measured functional residual capacity (FRC) and airway diameters of the model were adjusted to match measured baseline RL and EL [6]. For bronchoconstriction, the VDefs defined from PET (fig. 1) were mapped into the model. The PET images typically cover two-thirds of the lung while the 3-D model included the full airway tree. To account for this, model VDefs were expanded outside the imaged field so that the resulting fraction of lung volume occupied by VDefs within the model was equivalent and contiguous to that occupied by VDefs within the imaged lung.

For bronchoconstriction the level of airway narrowing applied to the model was defined based on whether or not the individual airway fed VDefs. First, a fixed fraction (40\%) of the

TABLE 1 Subject demographics

\begin{tabular}{|c|c|c|c|c|c|c|c|}
\hline Subject & Sex & Age & $\begin{array}{c}\text { Baseline upright } \\
\text { FEV } 1\end{array}$ & $\begin{array}{c}\text { Baseline upright FEV } 1 \\
\% \text { pred }\end{array}$ & $\begin{array}{l}\text { Baseline supine } \\
\text { FEV } 1\end{array}$ & $\begin{array}{c}\text { Post MCH supine } \\
\text { FEV } 1\end{array}$ & $\mathrm{PC}_{20} \mathrm{mg} \cdot \mathrm{mL}^{-1}$ \\
\hline 2 & M & 23 & 4.14 & 101 & 2.78 & 1.19 & 1.25 \\
\hline 3 & $\mathrm{~F}$ & 24 & 3.5 & 101 & 2.27 & 1.49 & 1.25 \\
\hline 4 & M & 57 & 1.77 & 53 & 1.41 & 0.59 & 0.05 \\
\hline 7 & M & 39 & 3.75 & 89 & 3.15 & 1.88 & 0.25 \\
\hline 8 & $\mathrm{~F}$ & 29 & 2.29 & 74 & 1.78 & 1.32 & 1.8 \\
\hline 9 & $\mathrm{~F}$ & 50 & 2.67 & 93 & 2.06 & 1.35 & 0.34 \\
\hline 10 & M & 40 & 3.81 & 91 & 3.3 & 2.22 & 0.25 \\
\hline Total & & $34 \pm 12$ & $3.18 \pm 0.82$ & $89.6 \pm 15.34$ & $2.4 \pm 0.58$ & $1.45 \pm 0.44$ & $0.69 \pm 0.62$ \\
\hline
\end{tabular}

Data are presented as $\mathrm{n}$ or mean \pm SEM. FEV1: forced expiratory volume in one second; \% pred: \% predicted; $\mathrm{MCH}$ : methacholine; $\mathrm{PC} 20$ : provocative dose causing a $20 \%$ fall in FEV1. 


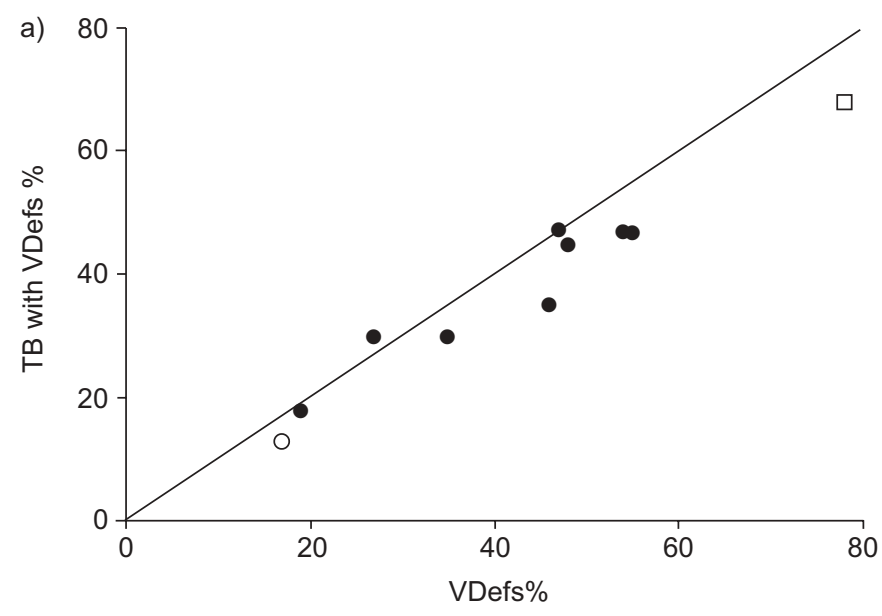

b)

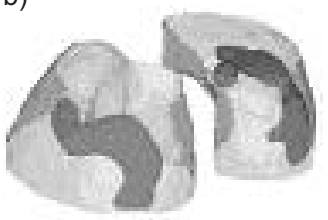

c)

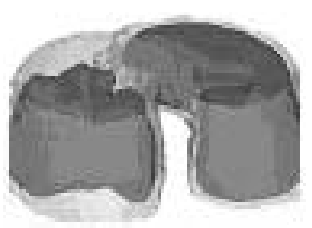

FIGURE 1. a) Relationship between the per cent of positron emission tomography (PET) imaged lung volume covered by ventilation defects (VDefs) and the per cent of terminal bronchi (TB) within the corresponding VDefs mapped into the three-dimensional model for each asthmatic subject. b, c) Exemplary threedimensional surface renderings of the PET-imaged lung field and VDefs (darker shading) corresponding to the two subjects with b) the smallest $(\bigcirc)$ and c) largest $(\square)$ extent of VDefs.

terminal bronchioles that led to VDefs were reduced in diameter to a fixed fraction $(20 \%)$ of that at baseline while the rest of the airway tree was left at the baseline size (pattern A). Next, in addition to the constriction conditions in pattern A, all bronchi exclusively feeding the VDefs were constricted to a fixed fraction (30\%) of baseline diameter (pattern B). The rationale for choosing the specific degrees of airway constriction in patterns $\mathrm{A}$ and $\mathrm{B}$ comes from a previous modelling study [5]. Finally, in addition to the constriction conditions in pattern B, heterogeneous constriction was imposed on the airways that did not lead to VDefs, by random sampling of Gaussian distributions covering a grid of means and SDs (pattern C).

The simulated lung mechanics best approaching those measured, for a given subject was taken as those yielding the lowest simulation index (SI) defined as:

$$
\mathrm{SI}=\frac{\sum_{i=1}^{n f}\left[\left(\operatorname{Re}_{m}-\operatorname{Re}_{d}\right)_{i}^{2}+\left(\operatorname{Im}_{m}-\operatorname{Im}_{d}\right)_{i}^{2}\right]}{\sum_{i=1}^{n f}\left(\operatorname{Re}_{d}^{2}+\operatorname{Im}_{d}^{2}\right)_{i}}
$$

where $\operatorname{Re}$ and Im are the real and imaginary part of lung impedance, respectively, $i$ denotes each of the $n f$ frequencies studied, d refers to experimental and $\mathrm{m}$ to modelled data. Therefore, the pair of mean and SD yielding a minimum value of SI (fig. 2) defined the constriction pattern for pattern C.

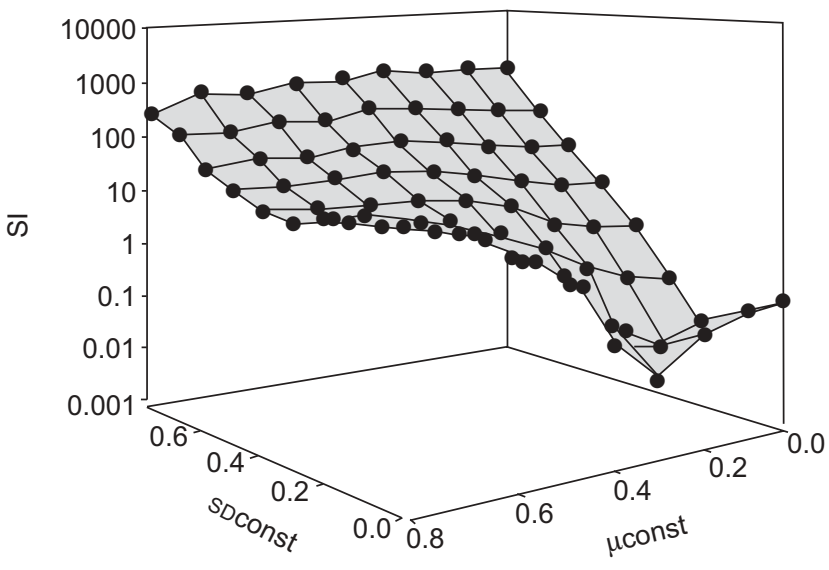

FIGURE 2. Simulation index (SI) plot presenting all simulations conducted with pattern C, for a typical subject. In each simulation constricted diameters of those airways feeding the regions outside the ventilation defects were defined by random draw of a constriction factor from a given Gaussian probability distribution. A unique minimum SI identifies the pair of mean ( $\mu$ const) and SD (SDconst) that provided best match between model and measured lung mechanics during bronchoconstriction.

\section{Statistical analysis}

Comparisons were performed using paired t-tests with significance level of $\mathrm{p}<0.05$. Linear regressions were conducted for baseline-normalised airway diameters $\left(D / D_{0}\right)$ and baseline-normalised airway conductance $(G / G)$ versus the extent of the model's VDefs. Linear regressions were also conducted for baseline-normalised airway conductance at $0.15 \mathrm{~Hz}$ during bronchoconstriction (GMch/GBase) versus the fraction of imaged lung volume covered by VDefs.

\section{RESULTS}

The sets of airway diameters of the model that simulated measured lung mechanics at baseline for all subjects, involved a substantial constriction with a small level of heterogeneity (mean \pm SD $0.42 \pm 0.08$ ). These adjusted airway diameters defined baseline conditions for each subject.

During bronchoconstriction all subjects showed a considerable increase in the frequency dependence and mean levels of $R \mathrm{~L}$ and EL compared with baseline (fig. 3). The severe reduction in terminal bronchiolar diameter, imposed on the model's VDefs (pattern A), caused only minor elevations in RL and EL from baseline that could not account for the obstructive changes in mechanics during bronchoconstriction (fig. 3). Additional constriction to all airways of the tree feeding the VDefs (pattern B) caused only a minor elevation in RL and EL relative to pattern A (fig. 3). However, additional heterogeneous reduction in diameters of airways feeding the rest of the lung (pattern C) substantially improved the match between simulated and measured values of RL and EL (fig. 3). As a result, there were systematic and significant reductions in SIs between patterns A and $B$ and between patterns $B$ and $C(p<0.05$; fig. 4).

It is therefore clear that, in addition to severe constriction of terminal bronchioles within VDefs and the airways leading to them, a certain degree of heterogeneous constriction needs to be applied to the rest of the airway tree to explain the changes in oscillatory mechanics measured in the subjects during bronchoconstriction. There was substantial variability in the 

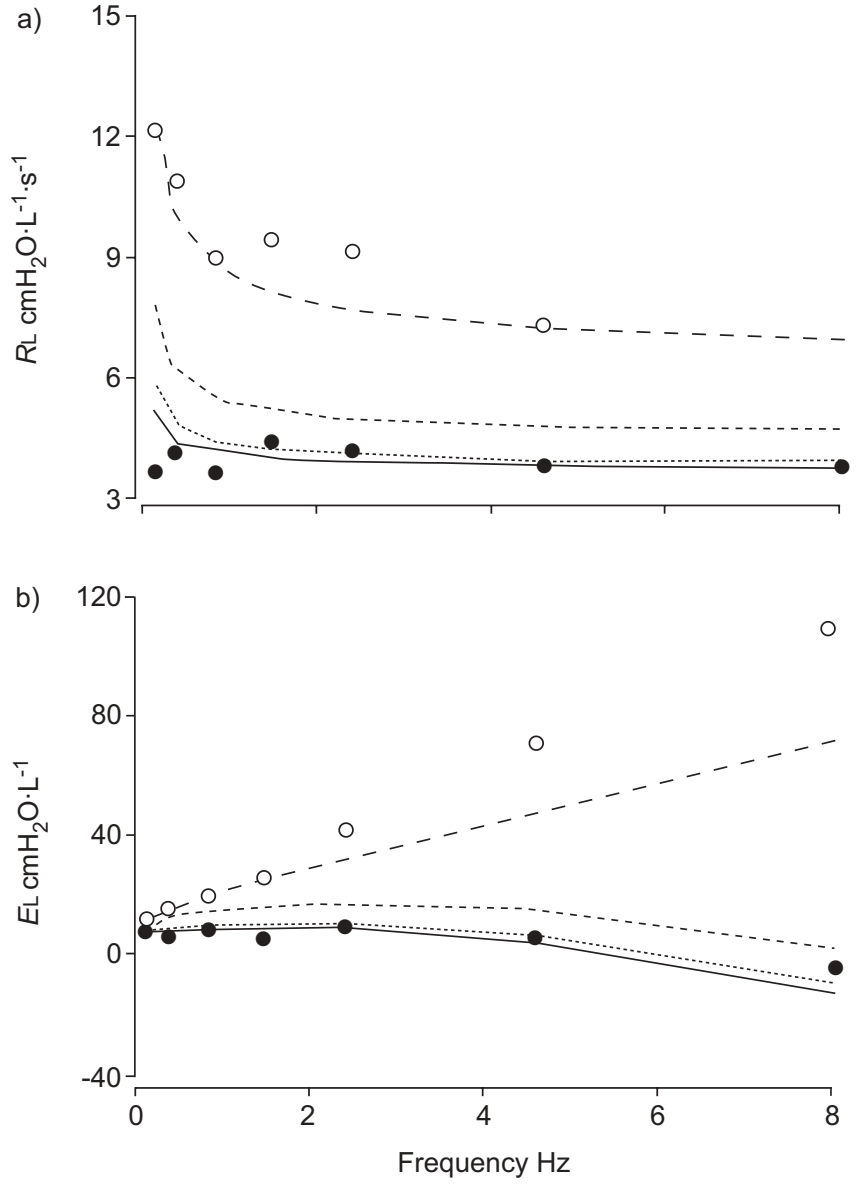

FIGURE 3. Typical plots of a) lung resistance $(R \mathrm{~L})$ and b) lung elastance $(E \mathrm{~L})$ versus frequency during baseline $(\bullet)$ and bronchoconstriction $(O)$ conditions. At baseline conditions $R L$ and $E L$ show small frequency dependence. As a result, the model simulations $(-)$ that best matched the measured lung mechanics at baseline involved only minor degrees of heterogeneous airway constriction. During bronchoconstriction, the mean levels and the frequency dependence of $R L$ and $E L$ were substantially elevated compared with those measured at baseline. Model simulations with only severe constriction of $40 \%$ (pattern $A ; \cdots \cdots$.$) ) terminal$ bronchioles within the mapped ventilation defects (VDefs), and in conjunction mild constriction only of airways leading to the VDefs (pattern B; - - -) could account for a small part of the measured changes in mechanics caused by bronchoconstriction. In contrast, the best match simulation with pattern C (-- ), which also involved heterogeneous reduction in the diameter of airways that did not feed the VDefs, was able to better approach the mechanics measured during bronchoconstriction.

extent of VDefs amongst subjects, ranging $19-80 \%$ of the imaged lung (fig. 1). This variability was not associated with the level or heterogeneity in constriction of airways not feeding VDefs (fig. 5). However, the average of $D / D$ o for all airways in each of the subject-specific models was negatively correlated $(r=-0.91)$ with the relative extent of the model's VDefs and can fit linear regression as:

$$
D / D_{o}=0.9012-0.38 \mathrm{~TB}
$$

where TB is the fraction of terminal bronchioles encompassed within the VDefs (fig. 6a).

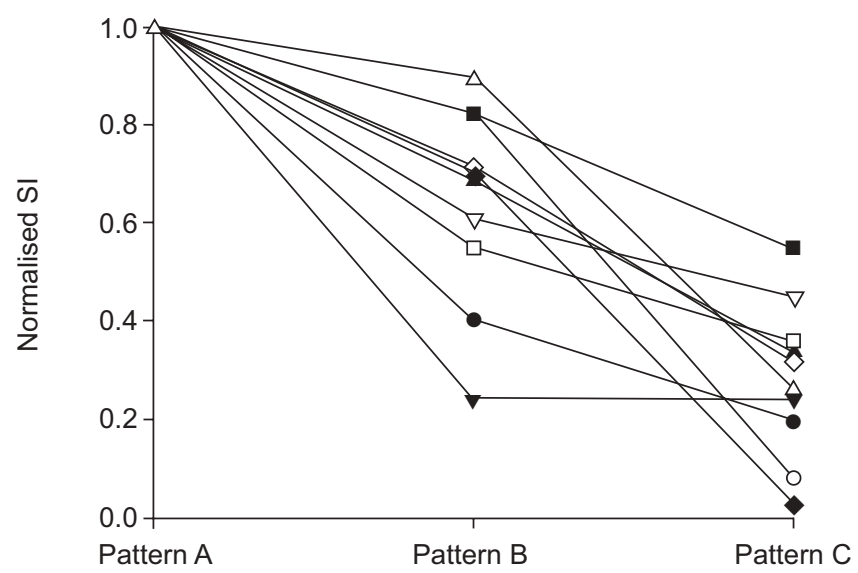

FIGURE 4. Improvement in simulation indices (SI) for input patterns applied to patterns $\mathrm{B}$ and $\mathrm{C}$ relative to those applied to pattern $\mathrm{A}$. The $\mathrm{SI}$ for each subject for patterns in $\mathrm{B}$ and $\mathrm{C}$ are normalised by the corresponding $\mathrm{SI}$ obtained for constriction with pattern A. All model simulations involved severe constriction of $40 \%$ of the terminal bronchioles within the mapped ventilation defects. Note the systematic reduction in $\mathrm{SI}$ (closer match in mechanics) between input patterns applied to patterns $\mathrm{B}$ and $\mathrm{C}$. $\mathrm{p}<0.05$ comparing $\mathrm{A}$ and $\mathrm{B} ; \mathrm{p}<0.001$; comparing input patterns applies to patterns $B$ and $C$. Each symbol represents an individual subject.

The assumption of an average $G / G$ for the model as proportional to $D / D_{0}$ to the fourth power $\left(D / D_{0}^{4}\right)$, also yielded a negative correlation $(r=-0.91)$ between $G / G$ o and $T B$ which can be approximated by linear regression as:

$$
G / G_{0}=0.60-0.68 \mathrm{~TB}
$$

This relationship (fig. 6) was consistent with the linear regression between a $G / G o$, measured during methacholineinduced bronchoconstriction (GMch/GBase), and the fraction of imaged lung volume encompassed by VDefs in the PET images $(\mathrm{r}=-0.48)$ :

$$
(\text { GMch } / \text { GBase })=\frac{1 / R_{\text {Mch }}}{1 / R_{\text {Base }}}=0.549-0.36 \text { VDef }
$$

where RMch and RBase are oscillatory lung resistance measured at $0.15 \mathrm{~Hz}$ during bronchoconstriction and at baseline, respectively, and VDef is the fraction of voxels of imaged lung encompassed within the VDefs (fig. 6b). The correlation was substantially reduced $(\mathrm{r}<0.001)$ when $\mathrm{GMch} / \mathrm{GBase}$ was used at $8 \mathrm{~Hz}$.

\section{DISCUSSION}

The main findings of the current study were as follows. 1) Severe constriction of terminal bronchioles and airways leading to VDefs was not enough to explain the mechanical obstruction measured in bronchoconstriction. 2) A significant degree of heterogeneous constriction of the rest of the airway tree was necessary to account for the measured changes in oscillatory mechanics of each subject. 3) Inter-subject differences in the average degree of constriction of airways not leading to VDefs were not correlated with the extent of VDefs.

Before discussing these findings, it is important to point out methodological assumptions and limitations of the current 


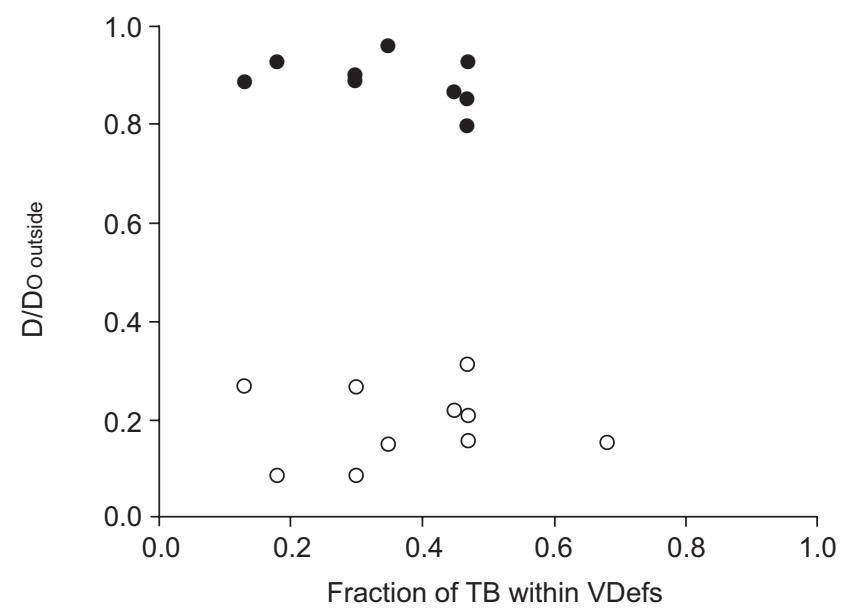

FIGURE 5. Plot of the average $(\bullet)$ and SD $(\bigcirc)$, of baseline-normalised airway diameter $\left(D / D_{0}\right.$ outside) of branches in the model feeding terminal units outside ventilation defects (VDefs) for the best match constriction pattern C. Data for each subject is plotted as a function of the fraction of total terminal bronchioles (TB) encompassed within the mapped VDefs. Both the degree and heterogeneity of the best match constriction patterns were similar amongst subjects and not correlated with the extent of the subject's VDefs.

methodology. Baseline airway diameters of the model were initially adjusted to match measured baseline RL and EL. Initially, a previously published model of airway dimensions [13] derived from a generic normal lung in the upright position for baseline conditions was used, as a result, the airway diameters had to be adjusted to account for the airway size differences between that generic model and the lung of each asthmatic subject studied. The actual amount of adjustment is also justified because the subjects were studied when lying down, a condition expected to reduce lung volume at FRC. Thus, it can be expected that to match the baseline oscillatory mechanics, the generic model required a significant mean level of constriction (mean 0.42). Interestingly, the SD of the adjustment was low (0.08) and thus represented a relatively homogenous constriction pattern.

Regions of VDefs, identified from PET images, were mapped on to an anatomically consistent 3-D model of the lungs. In this model, severe constriction of the terminal bronchioles of as much as $20 \%$ of the baseline diameter was defined because previous modelling analysis [5] showed that at least that much constriction was required to simulate the elevation of elastance at normal breathing frequencies observed in asthmatics during bronchoconstriction. Terminal bronchioles in the model were not fully obstructed because analysis of the PET images showed that units within VDefs ventilated slowly but were usually not totally unventilated. Preliminary analysis of the data also showed that such a reduction in diameter resulted in regional ventilation of VDefs, which was consistent with that observed in poorly ventilated units with PET $[3,4,14]$.

Previous analysis of the PET images has shown that voxels within the VDefs were not uniformly hypoventilated but instead had a mixture of ventilating units and hypoventilating units $[4,15]$. To account for this it was assumed that $40 \%$ of terminal bronchioles within the VDefs of the model were severely constricted while the diameters of the rest were kept at baseline levels. No significant differences in the impedance spectrum predicted by the model were observed when as much as $100 \%$ of the terminal bronchioles within the VDefs of the model were severely constricted. Diameters of the airways feeding VDefs were reduced to $30 \%$ of baseline consistent with previous modelling results [6]. The simulations showed that exclusive constriction to $\leqslant 30 \%$ of baseline in airways leading

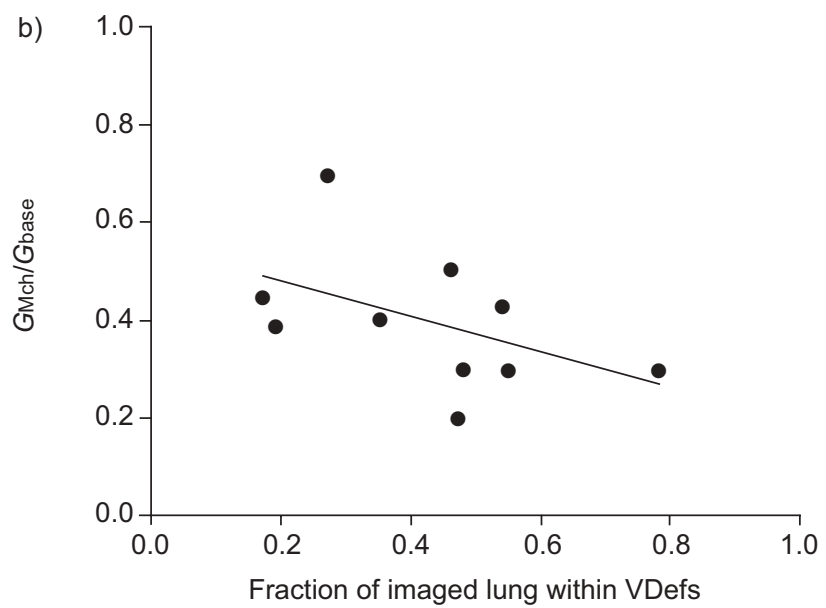

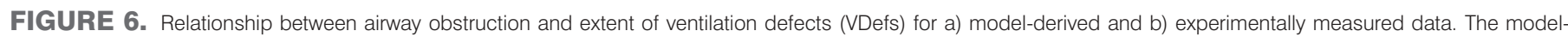

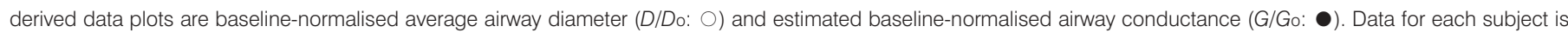

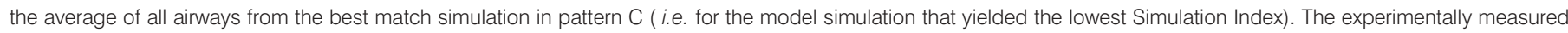

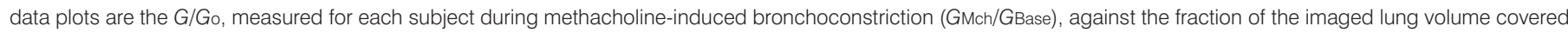

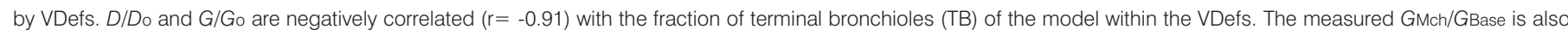

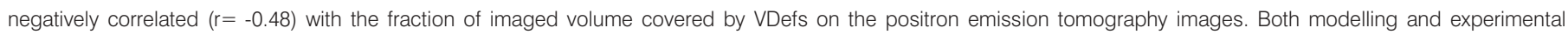

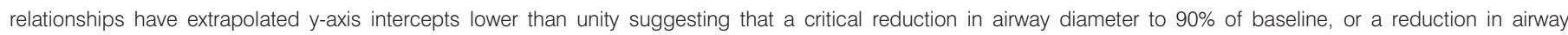
conductance to $60 \%$ of baseline, needs to occur before sizable VDefs develop in the lungs. 
to VDefs had a minimal effect on elevating RL and EL. Heterogeneous constriction of airways outside the VDefs was defined by random sampling of constriction factors from Gaussian probability distributions [7]. The constricted diameter of each airway was constrained to be $\geqslant 20 \%$ and $\leqslant 110 \%$ of its baseline diameter. Airway diameters as large as $110 \%$ of baseline were allowed since airway dilation, possibly by increased parenchymal tethering forces, had been visualised with computed tomography (CT) in certain airways during bronchoconstriction [16, 17]. However, the minor fractional changes in diameter introduced with this assumption had only minimal effect on the simulated oscillatory mechanics. Introducing the above constraints resulted in distributions of final airway diameters with mean and SDs that were different from those defining the original constriction factor Gaussian distributions. The current study presents the means and SDS of the airway diameters actually imposed on the model.

\section{Integration of ventilation imaging and lung mechanics}

Topographical information from PET images was synthesised into a 3-D model of the airway tree and used to test the effects of regional changes in airway diameter on lung mechanics. Consistent with previous studies [6] it was found that most of the frequency dependence of $R \mathrm{~L}$ and EL after bronchoprovocation could only be accounted for if heterogeneous constriction was added to the airway tree.

Both the mean and SD of constriction in the best match simulations were similar amongst subjects and uncorrelated with the extent of their respective VDefs (fig. 5). In other words, the degree of heterogeneous constriction of the rest of the tree appeared independent of the extent of the VDefs. This constriction corresponded to an average airway diameter of $\sim 90 \%$ of baseline. The specific value of such constriction could have been affected by various model assumptions. The present study assumed that all airways distal to the third generation were submitted to a similar degree of heterogeneous constriction. This was assumed because cartilaginous protection decreases rapidly for airways distal to that generation. To the current authors' knowledge, there is no experimental evidence showing preferential constriction of airways of a given set of generations proximal to the terminal bronchioles. Instead, direct imaging of bronchoconstricted airways of animals [18] and humans $[16,19]$ has shown heterogeneous reductions in airway diameter at all levels of the tree within the imaging resolution of $\mathrm{CT}(>2 \mathrm{~mm})$.

The best match simulations yielded a negative correlation between the average baseline-normalised airway diameters and the extent of the VDefs among subjects. This finding implies that part of the differences in the global mechanical response among subjects (fig. 6) could be explained by differences in the extent of their respective VDefs and, thus, by the number of severely constricted terminal units.

The inverse relationship between mean baseline-normalised diameter of all airways of the model and the fraction of terminal bronchi involved in the VDefs was consistent with the equivalent relationship for experimental data obtained between the baseline-normalised airway conductance, measured at $0.15 \mathrm{~Hz}$, and the fraction of the imaged lung involved in VDefs.
Both modelling and experimental correlations can be expected given the concentration of severely constricted terminal airways in the VDefs from the modelled and imaged lungs. A less expected finding was that both modelling and experimental relationships (fig. 6) had y-axis intercepts that were lower than unity ( $G$ reduction for VDefs of negligible size). In fact, the y-intercept of the experimental relation (0.60) and that for the model generated relation (0.52) were quite similar. This suggests that a critical reduction of conductance of as much as $40-50 \%$ may take place before a patchy pattern of ventilation distribution develops.

$G$ calculated as the inverse of $R \mathrm{~L}$ is usually regarded as a surrogate for changes of the airway lumen. Both $G$ and $R L$ are typically measured at relatively high frequencies $(5-8 \mathrm{~Hz})$ because of the minimal contribution of tissue resistance and parallel heterogeneity exhibited at these frequencies as previously depicted by network models of lung mechanics using rigid airways. When using models with rigid airways, heterogeneity is in fact more of a contributor to the low frequency data $(0.15 \mathrm{~Hz})$ compared with the high frequency data $(8 \mathrm{~Hz})$ [20]. To test whether in realistic conditions the presence of airway compliance could affect that conclusion, G/ Go was also estimated at $8 \mathrm{~Hz}$ assuming rigid walls with the constriction conditions found to best match the measured changes in mechanics during bronchoconstriction for each subject. If $G / G_{0}$ at $8 \mathrm{~Hz}$ for the rigid airways is taken as the best indicator of changes in average airway lumen during bronchoconstriction, then the deviation between this and G/Go derived with compliant airways, at any frequency, could be considered as the error in assessing the change in airway lumen under realistic conditions at that frequency. It was found that, in the models with compliant airways, G/Go at $8 \mathrm{~Hz} \quad\left(\right.$ mean $\pm \mathrm{SD} \quad 0.15 \pm 0.13 \mathrm{~L}^{-1} \cdot \mathrm{cmH} 20 \cdot \mathrm{s}^{-1}$ ) deviated three times more than $G / G$ at $0.15 \mathrm{~Hz} \quad\left(0.053 \pm 0.0408 \mathrm{~L}^{-1}\right.$. $\mathrm{cmH} 20 \cdot \mathrm{s}^{-1}$ ) relative to $\mathrm{G} / \mathrm{Go}$ at $8 \mathrm{~Hz}$ obtained assuming rigid airways: this was true in the models of nine out of 10 subjects. It is concluded, due to the presence of airway compliance, that the change in global $G$ measured at a breathing frequency of $0.15 \mathrm{~Hz}$ can better approximate the change in airway calibre than when measured at $8 \mathrm{~Hz}$. This may explain the better correlation obtained in figure 6 when $G / G_{0}$ is plotted for low rather than high frequency.

The suggestion that a critical reduction of conductance takes place before a patchy pattern of ventilation distribution develops is consistent with the behaviour of the network model of the airway tree [4]. This network model is predictive of the formation of the VDefs and change in airway diameter as a result of dynamic interactions among different airways of the tree unlike the 3-D airway tree model, which is not predictive of the formation of the VDefs, and requires airway diameters and the location of ventilation defects as inputs. More specifically, the network model incorporated the effects of tidal stretch on smooth muscle [21, 22], the interdependence between airway wall and parenchymal forces [23, 24] and the interdependent behaviour between airways within a branching tracheobronchial tree [25]. This model was assumed to be virtually symmetrical and with uniform structural and functional properties to demonstrate that heterogeneity in those properties was not a requirement to develop the heterogeneity in constriction observed in asthma. Indeed, the model showed 
that progressive increases in smooth muscle tone, up to a critical level, resulted in progressive but uniform airway constriction. However, smooth muscle activation beyond that critical level led to a very heterogeneous pattern of ventilation and airway obstruction with large clusters of constricted terminal bronchioles [4] equivalent to large VDefs observed with PET [15]. Further activation of smooth muscle in the model expanded the extent of the VDefs as well as the number of severely constricted terminal bronchioles within them, while the rest of the lung remained uniformly ventilated. The findings of the present study are consistent with the following predictions of that theoretical model [4]. 1) Exclusive constriction of airways leading to ventilation defects, whether peripheral or more central was not enough to explain the obstructive changes in lung mechanics measured in the asthmatic subjects. To explain those changes, a certain degree of heterogeneous airway constriction to the rest of the airway tree was also required. 2) Although the differences in mechanical response to bronchoconstriction amongst different subjects were associated with differences in the extent of their VDefs, the constriction of airways leading to the rest of the lung was not associated with the inter-subject differences in the extent of the VDefs. In other words, it appears that once VDefs start to form, further smooth muscle constriction increases the relative extent of the VDefs but not the degree of constriction of the airways leading to the rest of the lung. 3) A non-unity y-axis intercept between the baseline-normalised conductance and the relative extent of VDefs is consistent with the prediction that a critical level of airway narrowing needs to be reached before sizable ventilation defects are developed.

In contrast to the highly uniform distribution of ventilation predicted by the theoretically symmetrical network model for regions outside the VDefs [4], the mechanical model in the present study showed that to explain the measured changes in oscillatory mechanics heterogeneity had to be present in airways that did not lead to VDefs. This is also consistent with results of regional analysis of the ventilation PET images [15]. This result is to be expected because the asymmetry in structural and functional properties intrinsic to the asthmatic lung.

The present study demonstrates that in addition to the largescale ventilation heterogeneity caused by clustering of severely constricted distal airways in VDefs [4], an average degree and heterogeneity of constriction in the rest of the airway tree was required to explain the measured changes in mechanical obstruction. Such heterogeneity was similar among the subjects studied and was not related to the extent of the VDefs. However, differences in mechanical response amongst patients could be attributed, in part, to the extent of the lung involved in ventilatory defects for each patient. These findings are consistent with predictions of a recent integrative computational model of a constricting lung [4] in which smooth muscle activation above a critical level resulted in clusters of highly constricted distal airways and a certain level of constriction of the rest of the tree, albeit to a much lesser extent than that of airways leading to VDefs. Both experiments and modelling suggest that for smooth muscle activation exceeding that critical level, VDefs increase in their extent while the rest of the airway tree does not constrict any further.

\section{Conclusion}

The presence of ventilation defects appears to explain not only the gas exchange impairment [3] during bronchoconstriction but also, in combination with a ventilation defects sizeindependent level of heterogeneous constriction of the rest of the airway tree, the increased pressure losses and mechanical impairment in asthma. Finally, the current results support the concept that the heterogeneous and patchy distribution of ventilation in asthma is a manifestation of a complex behaviour of the system rather than the independent behaviour of individual airways.

\section{REFERENCES}

1 Samee S, Altes T, Powers P, et al. Imaging the lungs in asthmatic patients by using hyperpolarized helium-3 magnetic resonance: assessment of response to methacholine and exercise challenge. J Allergy Clin Immunol 2003; 111: 1205-1211.

2 Altes TA, Powers PL, Knight-Scott J, et al. Hyperpolarized 3He MR lung ventilation imaging in asthmatics: preliminary findings. J Magn Reson Imaging 2001; 13: 378-384.

3 Marcos F, Vidal Melo MF, Harris RS, Layfield JD, Venegas JG. Topographic basis of bimodal ventilationperfusion distributions during bronchoconstriction in sheep. Am J Respir Crit Care Med 2005; 171: 714-721.

4 Venegas JG, Winkler T, Musch G, et al. Self-organized patchiness in asthma as a prelude to catastrophic shifts. Nature 2005; 434: 777-782.

5 Lutchen KR, Gillis H. Relationship between heterogeneous changes in airway morphometry and lung resistance and elastance. J Appl Physiol 1997; 83: 1192-1201.

6 Tgavalekos NT, Tawhai M, Harris RS, et al. Identifying airways responsible for heterogeneous ventilation and mechanical dysfunction in asthma: an image-functional modeling approach. J Appl Physiol 2005; 99: 2388-2397.

7 Tgavalekos NT, Venegas JG, Suki B, Lutchen KR. Relation between structure, function, and imaging in a threedimensional model of the lung. Ann Biomed Eng 2003; 31: 363-373.

8 Lutchen KR, Yang K, Kaczka DW, Suki B. Optimal ventilation waveforms for estimating low-frequency respiratory impedance. J Appl Physiol 1993; 75: 478-488.

9 Kaczka DW, Ingenito EP, Israel E, Lutchen KR. Airway and lung tissue mechanics in asthma. Effects of albuterol. Am J Respir Crit Care Med 1999; 159: 169-178.

10 Musch G, Layfield JD, Harris RS, et al. Topographical distribution of pulmonary perfusion and ventilation, assessed by PET in supine and prone humans. J Appl Physiol 2002; 93: 1841-1851.

11 Crapo RO, Casaburi R, Coates AL, et al. Guidelines for methacholine and exercise challenge testing-1999. This official statement of the American Thoracic Society was adopted by the ATS Board of Directors, July 1999. Am J Respir Crit Care Med 2000; 161: 309-329.

12 Kuwano K, Bosken CH, Pare PD, Bai TR, Wiggs BR, Hogg JC. Small airways dimensions in asthma and in chronic obstructive pulmonary disease. Am Rev Respir Dis 1993; 148: 1220-1225.

13 Tawhai MH, Hunter P, Tschirren J, Reinhardt J, McLennan G, Hoffman EA. CT-based geometry analysis 
and finite element models of the human and ovine bronchial tree. J Appl Physiol 2004; 97: 2310-2321.

14 Venegas JG, Schroeder T, Harris S, Winkler RT, Melo MF. The distribution of ventilation during bronchoconstriction is patchy and bimodal: a PET imaging study. Respir Physiol Neurobiol 2005; 148: 57-64.

15 Harris RS, Winkler T, Tgavalekos N, et al. Regional pulmonary perfusion, inflation, and ventilation defects in bronchoconstricted patients with asthma. Am J Respir Crit Care Med 2006; 174: 245-253.

16 Brown RH, Scichilone N, Mudge B, Diemer FB, Permutt S, Togias A. High-resolution computed tomographic evaluation of airway distensibility and the effects of lung inflation on airway caliber in healthy subjects and individuals with asthma. Am J Respir Crit Care Med 2001; 163: 994-1001.

17 Kotaru C, Coreno A, Skowronski M, Muswick G, Gilkeson RC, McFadden ER Jr. Morphometric changes after thermal and methacholine bronchoprovocations. J Appl Physiol 2005; 98: 1028-1036.
18 Brown RH, Mitzner W. The myth of maximal airway responsiveness in vivo. J Appl Physiol 1998; 85: 2012-2017.

19 King GG, Salome CM. Multimodality measurements of small airways disease. Eur Respir J 2006; 27: 250-252.

20 Kaczka DW, Ingenito EP, Suki B, Lutchen KR. Partitioning airway and lung tissue resistances in humans: effects of bronchoconstriction. J Appl Physiol 1997; 82: 1531-1541.

21 Fredberg JJ. Bronchospasm and its biophysical basis in airway smooth muscle. Respir Res 2004; 5: 2.

22 Gunst SJ, Stropp JQ. Pressure-volume and length-stress relationships in canine bronchi in vitro. J Appl Physiol 1988; 64: 2522-2531.

23 Anafi RC, Beck KC, Wilson TA. Impedance, gas mixing, and bimodal ventilation in constricted lungs. J Appl Physiol 2003; 94: 1003-1011.

24 Anafi RC, Wilson TA. Airway stability and heterogeneity in the constricted lung. J Appl Physiol 2001; 91: 1185-1192.

25 Weibel ER. Morphometry of the Human Lung. Berlin, Springer, 1963; pp. 115-126. 